\title{
25 Research Soure \\ Deep Polar Residual Network for Identifying Myocardial Infarction in Mce Imaging
}

\section{Wenqian Shen}

Second Affiliated Hospital of Harbin Medical University

Jingyi Xue

First Affiliated Hospital of Harbin Medical University

\section{Yanhui Guo}

University of Illinois at Springfield

Haiyuan Yu

Second Affiliated Hospital of Harbin Medical University

\section{Shuang Chen}

Second Affiliated Hospital of Harbin Medical University

\section{Yan Wu}

Second Affiliated Hospital of Harbin Medical University

\section{Guoqing Du ( $\nabla$ duguoqing9@163.com )}

Department of Ultrasound, The Second Affiliated Hospital of Harbin Medical University, Harbin, 150086 China; https://orcid.org/0000-0001-7963-4652

\section{Research}

Keywords: Myocardial infarction, Myocardial contrast echocardiography, Deep learning model, Polar system

Posted Date: September 25th, 2020

DOI: https://doi.org/10.21203/rs.3.rs-80044/v1

License: (1) (1) This work is licensed under a Creative Commons Attribution 4.0 International License. Read Full License 


\section{Abstract}

Background: Ultrasonic diagnosis of myocardial infarction (MI) varies markedly among radiologists, and is also time-consuming. In addition, there is a high level of inter-observer variation in diagnosis of $\mathrm{Ml}$. The purpose of this study is to develop a deep learning-based model that automatically recognizes $\mathrm{Ml}$ in myocardial contrast echocardiography(MCE) images, and to compare the model performance against radiologists with varying experience in echocardiography.

Methods: In this study, a polar residual network (PResNet) model was employed to recognize MI segments in MCE images of Sprague-Dawley (SD) rats. All SD rats were randomly divided into the MI model group $(n=15)$ and the sham group $(n=10)$. Rats in MI model group were infarcted via left anterior descending coronary artery ligation. Ultimately, MCE was performed on SD rats to observe their myocardium. Left ventricle (LV) short-axis views of MCE at the basal and midpapillary muscle levels for each SD rat were divided into 6 segments separately. The PResNet model and six radiologists each diagnosed 276 MCE image segments. Masson's trichrome staining was used as the gold standard for MI presence or absence. The PResNet model and radiologist diagnostic performance were evaluated using sensitivity, specificity, and area under the receiver operating characteristic curve (AUC). Furthermore, analysis duration per rat and interobserver variability were also compared.

Results: Masson's trichrome stain revealed 30 infarcted segments out of the 276 total segments. The PResNet model achieved an AUC of 0.89 (0.85-0.93), which was comparable to that of senior radiologists $(0.89 ; 0.85-0.93)$. Relative to senior radiologists, the PResNet model presented slightly lower sensitivity but significantly higher specificity. The mean sensitivity and specificity for the junior radiologists were significantly lower than both the PResNet model and the senior radiologists. In terms of analysis duration, the PResNet model (148-169sec) was markedly faster than both junior (314-366sec) and senior (193$235 \mathrm{sec})$. Moreover, the intrasample agreement for the PResNet model $(k=0.96)$ was better than the interobserver agreement for the junior $(k=0.81)$ and senior radiologists $(k=0.86)$.

Conclusion: The PResNet model not only improved diagnostic performance and observer consensus but also improve the diagnose speed. It is a promising tool for identifying MI.

\section{Introduction}

Myocardial infarction (MI) threatens thousands of patients with coronary heart disease (CHD) worldwide every year [1]. Three-year mortality rates following MI range from $5 \%$ to $10 \%$, with the overall rate of major adverse cardiac events reaching as high as $30 \%$ [2]. The formation of an occlusive thrombus within a coronary artery leads to cardiac ischemia and infarction. If myocardial cell necrosis, dissolution, and myocardial microvascular perfusion disorder are not treated promptly, infarction size expansion occurs, further damaging left ventricular (LV) function and resulting in an irreversible loss of myocardial contractility and initiation of negative remodeling [3]. Eventually, patients may be at risk of interventricular septum perforation, acute heart failure, and free ventricular wall rupture. Since appropriate treatment 
needs to be delivered during the early stages of infarction, accurate and timely identification of $\mathrm{Ml}$ is paramount.

Echocardiography provides a real-time visualization way of the regional wall motion abnormalities associated with myocardial ischemia and is generally used for CHD diagnosis and prognosis assessment for [4]. Several new echocardiographic technologies have been developed to improve MI detection capabilities, such as speckle-tracking echocardiography [5], 3-D echocardiography [6], and myocardial contrast echocardiography (MCE) [7]. In particular, MCE not only clearly shows the presence of wall motion abnormalities, but also assesses the degree and adequacy of microvascular perfusion. Hence, MCE is an ideal tool for identifying the extent of myocardial infarction, with both prognostic and therapeutic implications [8].

Echocardiogram interpretation can vary significantly between operators. Besides, analyzing large amounts of imaging data is labor-intensive and time-consuming [9]. At present, MCE is capable of making semi-quantitative assessments. However, this process remains limited by human error in data acquisition and interpretation. Machine learning (ML), a subfield of artificial intelligence, has advantages to solve these problems. It involves training a computer to develop comprehensive algorithms through the analysis of large amounts of data rapidly, consistently, and accurately. ML models have been used in many aspects of echocardiography, including view classification [10-12], automated measurements [1318], automated valve disease assessment [19-22], and the classification of pathological patterns [23, 24]. Nonetheless, the application of ML models for the detection of MI using echocardiography is still in its infancy. Many classical ML models rely on describing new myocardium features-this requires manual tracing and multiple trials, thus limiting performance and generalization [25-27]. Deep learning, a type of artificial neural network, can automatically process larger and more complex data sets using technologies such as convolutional neural networks. It has been applied to the diagnosis of thyroid nodules [28], as well as the detection of breast tumors [29, 30] and skin cancers [31], and might be capable of addressing current limitations in ML for MI analysis.

Our team has been working on a computer aided diagnosis (CAD) approach to evaluating myocardial perfusion and infarct size using MCE [8]. Current deep learning approaches used original images presenting rectangular shapes. However, the myocardium in the short-axis view is annular, and thus DL approaches suited for rectangular images do not describe radial information well. To overcome this drawback, we proposed a polar residual network (PResNet) to identify MI regions in MCE images, with a new polar layer designed to transfer MCE images into the polar system. The current study sought to examine the application of PResNet for accurately detecting and diagnosing infarcts in MCE images. Following the initial success in examining MCE segments obtained from infarcted and sham-operated Sprague-Dawley (SD) rats, we then looked at the application of the PResNet model for clinic diagnosis, comparing the diagnostic performance of PResNet with that of radiologists with varying levels of experience.

\section{Methods}




\section{Animal model experimental protocols}

Male SD rats $(220-250 \mathrm{~g})$ were supplied by the Experimental Animal Center at the Second Affiliated Hospital of Harbin Medical University. Animal protocols were approved by the Institutional Animal Care Committee of Harbin Medical University, Heilongjiang Province, China. All experiments followed the Guide for the Care and Use of Laboratory Animals published by the U.S. National Institutes of Health (NIH Publication 85-23, revised 1996).

All SD rats were randomly divided into either the MI model group $(n=15)$ or the sham group $(n=10)$. Myocardial infarction was induced in MI model group rats via permanent ligature of the left anterior descending coronary artery, while sham group rats underwent the same operational procedure without artery ligation. All rats were anesthetized with $10 \%$ chloral hydrate and fixed on an operating table. After anesthetic induction, the rats were mechanically ventilated with a positive pressure ventilator. Chest hair was then removed and a thoracotomy was performed between the third and fourth intercostal spaces to expose the whole heart. The left anterior descending artery was isolated and ligated at about $2 \mathrm{~mm}$ on the lower edge of the left auricle using a 7.0 silk snare. Whitening of the cardiac apex provided a visual indication of successful ligation. The chest was then sutured and closed. After resuscitation, the trachea cannula was removed, and all rats were returned to the animal housing facility with free access to food and water. MCE videos were collected one week post-surgery.

\section{MCE data acquisition}

MCE was performed using an ultrasound scanner (Vivid 7, GE Healthcare, Milwaukee, WI, USA) equipped with a $10 \mathrm{~S}$ transducer $(8-10 \mathrm{MHz})$. Rats were anesthetized using the above method and placed on the examination bed. The ultrasound mechanical index setting was set to 0.2 , and time gain compensation and 2-D gain settings were adjusted to suppress any nonlinear signals from the tissue before contrast agent infusion. SonoVue (Bracco, Milan, Italy) was then infused into the tail vein at a rate of $1.2 \mathrm{~mL} / \mathrm{h}$. MCE images were digitally acquired, starting at peak myocardial opacity until the disappearance of contrast from the myocardium. After imaging, rats were euthanized under deep anesthesia.

Electrocardiogram changes were continuously monitored using an electrocardiograph throughout the image acquisition process. The LV short-axis view MCE videos were obtained at the basal and midpapillary muscle levels, and digital data of 6-10 consecutive heart cycles were recorded on magnetooptical disks. The images showing the most favorable myocardial perfusion for each rat were selected analysis In this study, the LV basal and midpapillary muscle levels were divided into six segments according to the guidelines[32].

\section{Pathological staining}

Rat hearts were excised after death and fixed in $4 \%$ paraformaldehyde for histopathologic analysis. Each heart was cut into three thick slices along the long axis from the apex to base. After standard paraffin embedding, two 5- $\mu \mathrm{m}$-thick sections were obtained from each slice for Masson's trichrome staining. Sections were then imaged using light microscopy and abnormal staining segments were counted. Blue- 
stained myocardial regions were considered to be infarcted, while red staining indicated the presence of viable myocardium.

\section{Deep polar residual network}

In MCE short-axis images, myocardium radial characteristics are important indicators of myocardial properties. Therefore, it was better to utilize this characteristic and transfer MCE images from a Descartes system into a polar system for observing myocardial regions and comparing them with radial neighboring regions. This study proposes a novel approach, the use of a polar layer in a deep learning network. After transfering myocardium regions into the polar system, the myocardium ring was converted into a band which is suitable for deep learning network to analyze. The CAD system firstly created a region of interest $(\mathrm{ROI})$ on the original MCE image shaped like a disk including only the myocardium and chamber interior. The radius of this disk was set at 250 pixels in order to cover all myocardial regions. The ROI image is mapped onto the polar coordinates using a polar layer to obtain a polar ROI (PROI) image. Because the infarction region may be present at different angles and locations, the myocardium is usually divided into six equal segments. The PROI image was thus divided into six patches accordingly (Fig. 1).

After the polar layer, the segment patches are transferred from sector shape to a rectangular shape, which is then fed into subsequent convolutional layers for further processing. Here, infarction detection was converted to a task to categorize the segment patches within normal and abnormal groups. A deep residual network was designed to classify PROI image section patch images. The structure of the deep polar residual network (PResNet) consists of the polar layer, convolutional layers, softmax layer, residual component, and classification component. The connection between the polar layer and the following layers was not trainable.

Rather than building a model from scratch, we improved the architecture of a pre-designed ResNet model and refined its weighting for identifying new images. Known as transfer learning, this decreases the necessary time investment for training and enhances the generalization ability of the network. The proposed model adds the polar layer on a pre-trained ResNet-50 network, and a binary cross-entropy function was used as the loss function for classification. With the pre-train ResNet-50 model as a backbone, different layers were added to categorize segment images into normal and abnormal groups. The whole system and related image results are illustrated in Figure 2. The PResNet model analyzed all MCE images three times consecutively.

\section{Observer study}

Six radiologists with varying levels of experience in echocardiography (1 to 15 years), blinded to the experimental process and pathology results, analyzed all MCE images independently. Three of the six radiologists were junior individuals with less echocardiography experience $(1,2$, and 3 years, respectively), while the other three were senior members $(10,13$, and 15 years of experience, respectively). All radiologists graded each segment according to the wall motion score (WMS) and the degree of 
myocardial perfusion in the MCE. Semiquantitative-WMS scoring system is as follows: WMS $=1$, normal or hyperkinetic; $W M S=2$, hypokinetic (reduced thickening); WMS=3, akinetic (absent or negligible thickening, e.g., scar); WMS=4, dyskinetic (systolic thinning or stretching, e.g., aneurysm) [32]. The degree of myocardial perfusion was also scored similarly: 1 =normal perfusion, $2=$ subendocardial perfusion defect, and $3=$ transmural perfusion defect [33]. A segment was deemed to be infarcted when the sum of its two scores was equal to or greater than six. Segments that did not meet these criteria were deemed to be non-infarcted.

\section{Statistical analysis}

A receiver operating characteristic $(\mathrm{ROC})$ analysis was applied to assess the diagnostic accuracy of both the radiologists and the deep learning model. The empirical ROC curve was formed by connecting the set of data points generated from the different cut points. The area under the ROC curve (AUC) was also calculated as a metric to evaluate the method's performance. Operator classification sensitivity and specificity were calculated, as well as the analysis duration for junior radiologists, senior radiologists, and the proposed deep learning model per rat. Analysis time medians were compared using Friedman analysis with multiple comparison post-hoc testing (Bonferroni). Box and whisker plots were obtained to present analysis time distributions $A p$-value less than 0.05 was considered to indicate a statistically significant difference. Interobserver variability was assessed with Cohen's kappa coefficient (Table 1). The Medcalc statistical software (Version 19.0.4 Schoonjans, Frank) was used to take AUC comparison and $k$ statistics. Other statistical analyses were performed with the SPSS software (version 23 , Chicago, IL, USA).

\section{Results}

\section{Animal MCE imaging}

Of the 25 rats, 2 died on the first day after modeling. The remaining 23 SD rats were imaged using MCE, with a total of 276 segments captured for analysis.

\section{Deep residual network}

Segments containing infarcted myocardium detected by the model were marked both in the polar layer and on the LV short-axis view. If no infarct segment was detected, the polar view and the LV short-axis view remained unmarked. Model markup results are shown in Figure 3(A-D).

\section{Pathological staining}

Of 276 total segments, 30 presented blue Masson's trichrome staining, indicating infarction (Fig. 3E, F). A clear boundary was visible between blue-stained infarcted tissue and red-stained viable myocardium.

\section{Comparison of the proposed PResNet model against radiologist analysis}


The sensitivity, specificity, and area under the receiver operating characteristic curve (AUC) for both the six radiologists and the proposed PResNet are shown in Figure 4 and Table 2, respectively. Pathological results were used as the gold standard. The mean values for the junior and senior radiologists are also shown in Table 2. The PResNet model achieved an AUC of 0.89 (95\% Cl: $0.85,0.93)$, which is comparable $(p=0.842)$ to the mean AUC of the senior radiologists $(0.89,95 \% \mathrm{Cl}: 0.85,0.93)$. The mean AUC of the junior radiologists was 0.68 ( $95 \% \mathrm{Cl}: 0.63,0.74)$, significantly below that achieved by deep learning $(p \leq 0.001)$. The performance of five of the six individual radiologists was worse than that of the PResNet model, with the lowest AUC being 0.68 (95\% Cl: $0.62,0.73)$ and the highest AUC being 0.93 (95\% $\mathrm{Cl}: 0.89$, 0.96).

For the six radiologists, their sensitivity values ranged from $53.33 \%$ to $93.33 \%$. The sensitivity of PResNet was $83.33 \%$ (95\% Cl: $65 \%, 94 \%)$, which was a little lower than the mean values for the senior radiologists (86.67\%; 95\% Cl: 69\%, 96\%). This difference in sensitivity between the deep learning model and the senior radiologists was statistically significant ( $p \otimes 0.05)$. In terms of specificity, the radiologists ranged from $83.33 \%$ to $92.68 \%$. The specificity of the PResNet model was $95.53 \%$ (95\% Cl: $92 \%$, $98 \%$ ), which was

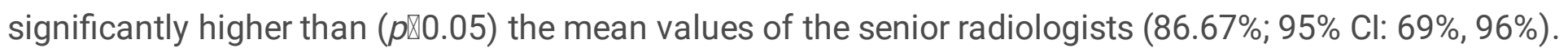
The mean sensitivity and specificity for the junior radiologists was $53.33 \%(95 \% \mathrm{Cl}: 34 \%, 72 \%)$ and $83.33 \%$ (95\% Cl: $78 \%, 88 \%$ ), respectively. Both of these values were significantly lower than those achieved by the deep learning model ( $p \bigotimes 0.001)$.

Total rat MCE image analysis duration for junior radiologists, senior radiologists, and the PResNet model is described in Table 3. Box and whisker plots (Fig. 5) were drawn to present the distribution of the time within the three groups. The median image analysis duration for the PResNet model was 154 seconds per rat (QR: 148, 169), which was markedly lower than both the junior radiologists (342 seconds per rat; QR: $314,366)$ and the senior radiologists (223 seconds per rat; QR: 193, 235).

\section{Interobserver variability}

Table 4 shows the level of agreement between observers for MI diagnosis. Agreement levels for both senior and junior radiologists were strong ( $k=0.86 ; k=0.81$, respectively). However, the agreement levels for the PResNet model's diagnoses were superior.

\section{Discussion And Summary}

Ultrasound diagnosis of $\mathrm{Ml}$ features have high levels of inter-observer variation. Furthermore, analyzing wall motion and myocardial perfusion using MCE images is time-consuming. To overcome these issues, automatic CAD based on ML has been introduced to this field. For example, a study by Sudarshan et al. [26] developed a new integrated index MI Index (MII) that takes into account multiple elements, including image pre-processing, the creation of two-dimensional textons, feature extraction from echocardiogram apical four chamber views, and feature ranking. This MII generated a single numerical value from which it was possible to discriminate between infarcted and normal myocardium. Sudarshan et al. [3] have also proposed Curvelet Transform and Local Configuration Pattern algorithms for the automatic 
distinguishing of $\mathrm{MI}$ severity using 2D echocardiograms. There, a composite index called the MI Risk Index (MIRI) was developed to detect normal, moderately, and severely infarcted myocardium. These studies analyzed apical four chamber view images from two-dimensional echocardiograms. However, previous studies, including the above stated, used original images containing rectangular images and failed to describe radial information and features well. Furthermore, MCE short-axis view images are better for displaying wall motion and the degree/extent of myocardial perfusion. We therefore developed a deep learning model, termed "PResNet", to recognize MI in LV short-axis view MCE images and compared the model's performance with radiologists with varying levels of experience in echocardiography.

Our proposed PResNet model showed similar diagnostic performance compared to senior radiologists with 10-plus years of experience. In particular, the biggest advantage of the PResNet model was the ability to provide superior infarct segment identification specificity. In terms of sensitivity, although the PResNet model could not reach the sensitivity of senior radiologists, it performed markedly better than junior radiologists. Indeed, our results indicated that junior radiologists-likely owing to their limited clinical experience-presented lower MI detection sensitivity and specificity than both the PResNet model and senior radiologists. As such, we believe that as junior radiologists gain experience-a process that cannot be accelerated-the short term usage of the PResNet model as an assistance tool can improve diagnostic accuracy. Also a note is that we found inconsistencies and variations in diagnosis between different radiologists for the same images. However, our deep learning model demonstrated strong repeatability, something that could compensate for this deficiency. Finally, visual MI diagnosis in the clinic is time-consuming and error-prone; the proposed PResNet model could reach diagnostic conclusions in a more rapid manner to save diagnostic time and improve consensus.

This study was not free from limitations. Namely, this study used rats, and we plan on moving towards larger mammals and MI patients in the future to see what adjustments may be required to accommodate the differences in cardiac architecture and MI presentation. Also, there were times in this study where MCE imaging quality was insufficient, and this issue will require further investigation.

In summary, the PResNet is a promising tool for identifying infarcted myocardium in MCE images, already capable of faster and more accurate $\mathrm{MI}$ segment identification relative to junior radiologists. As such, we believe that PResNet can help radiologists and physicians with expertise in echocardiography efficiently detect and intervene before MI progresses to worse outcomes. More studies will further validate our findings.

\section{Abbreviations}

AUC: Area under the receiver operating characteristic curve; CAD: Computer aided diagnosis; $\mathrm{Cl}$ : Confidence interval; LV: Left ventricle; MCE: Myocardial contrast echocardiography; MI: Myocardial infarction; ML: Machine learning; PResNet: Polar residual network; PROI: Polar ROI; ROC: Receiver operating characteristic; ROI: Region of interest; SD: Sprague-Dawley; WMS: Wall motion score. 


\section{Declarations}

\section{Acknowledgments}

We thank Dr. Nathan C. Ni for assistance with manuscript preparation and editing.

\section{Funding}

This study was supported by grants from the National Natural Science Foundation of China (81671762), the Postdoctoral Starting Foundation of Heilongjiang Province (LBH-Q16144), and the Graduate Research and Practice Innovation Project of Harbin Medical University (YJSSJCX2019-42HYD).

\section{Availability of data and materials}

The data that support the findings of this study are available on request from the corresponding author, Guoqing Du. The data are not publicly available due to the containing information that could compromise the privacy of research participants.

\section{Authors' contributions}

Shen WQ, Xue JY and Guo Y conceived of the presented idea. Shen WQ developed the theory and performed the computations. Shen WQ and Xue JY verified the analytical methods. Shen WQ wrote the paper under the supervision of Guo Y and Du GQ. All authors collected the data and discussed the final manuscript.

\section{Authors' information}

- mail addresses: 18254359537@163.com (Wenqian Shen), xuejingyi72@163.com (Jingyi Xue), gyh78@qq.com (Yanhui Guo), yhy0701a@163.com (Haiyuan Yu),393453418@qq.com (Shuang Chen), yanwu10240@163.com_(Yan Wu),_duguoqing9@163.com_(Guoqing_Du).

\section{Ethics approval and consent to participate}

Animal protocols were approved by the Institutional Animal Care Committee of Harbin Medical University, Heilongjiang Province, China. All experiments followed the Guide for the Care and Use of Laboratory Animals published by the U.S. National Institutes of Health (NIH Publication 85-23, revised 1996) and that such approvals are acknowledged within the manuscript.

\section{Consent for publication}

Not applicable.

\section{Competing interests}

The authors declare that they have no competing interests. 


\section{References}

1. Hu C, Tkebuchava T, Hu D. Managing acute myocardial infarction in China. Eur Heart J. 2019;40(15):1179-81. Epub 2019/04/15. doi: 10.1093/eurheartj/ehz182. PubMed PMID: 30982072.

2. Palasubramaniam J, Wang $X$, Peter K. Myocardial Infarction-From Atherosclerosis to Thrombosis. Arterioscler Thromb Vasc Biol. 2019;39(8):e176-e85. Epub 2019/07/25. doi:

10.1161/ATVBAHA.119.312578. PubMed PMID: 31339782.

3. Sudarshan VK, Acharya UR, Ng EY, Tan RS, Chou SM, Ghista DN. Data mining framework for identification of myocardial infarction stages in ultrasound: A hybrid feature extraction paradigm (PART 2). Comput Biol Med. 2016;71:241-51. Epub 2016/02/22. doi:

10.1016/j.compbiomed.2016.01.029. PubMed PMID: 26897481.

4. Alsharqi M, Woodward WJ, Mumith JA, Markham DC, Upton R, Leeson P. Artificial intelligence and echocardiography. Echo Res Pract. 2018;5(4):R115-R25. Epub 2018/11/08. doi: 10.1530/ERP-180056. PubMed PMID: 30400053; PubMed Central PMCID: PMCPMC6280250.

5. van Mourik MJW, Zaar DVJ, Smulders MW, Heijman J, Lumens J, Dokter JE, et al. Adding SpeckleTracking Echocardiography to Visual Assessment of Systolic Wall Motion Abnormalities Improves the Detection of Myocardial Infarction. J Am Soc Echocardiogr. 2019;32(1):65-73. Epub 2018/10/21. doi: 10.1016/j.echo.2018.09.007. PubMed PMID: 30340888.

6. Streiff C, Zhu M, Panosian J, Sahn DJ, Ashraf M. Comprehensive evaluation of cardiac function and detection of myocardial infarction based on a semi-automated analysis using full-volume real time three-dimensional echocardiography. Echocardiography. 2015;32(2):332-8. Epub 2014/06/17. doi: 10.1111/echo.12643. PubMed PMID: 24930502.

7. Ito H. Myocardial contrast echocardiography after myocardial infarction. Curr Cardiol Rep. 2012;14(3):350-8. Epub 2012/03/23. doi: 10.1007/s11886-012-0263-0. PubMed PMID: 22437372.

8. Du GQ, Xue JY, Guo Y, Chen S, Du P, Wu Y, et al. Measurement of myocardial perfusion and infarction size using computer-aided diagnosis system for myocardial contrast echocardiography. Ultrasound Med Biol. 2015;41(9):2466-77. Epub 2015/06/07. doi: 10.1016/j.ultrasmedbio.2015.04.012. PubMed PMID: 26048775.

9. Sudarshan V, Acharya UR, Ng EY, Meng CS, Tan RS, Ghista DN. Automated Identification of Infarcted Myocardium Tissue Characterization Using Ultrasound Images: A Review. IEEE Rev Biomed Eng. 2015;8:86-97. Epub 2014/05/08. doi: 10.1109/RBME.2014.2319854. PubMed PMID: 24803322.

10. Khamis H, Zurakhov G, Azar V, Raz A, Friedman Z, Adam D. Automatic apical view classification of echocardiograms using a discriminative learning dictionary. Med Image Anal. 2017;36:15-21. Epub 2016/11/07. doi: 10.1016/j.media.2016.10.007. PubMed PMID: 27816858.

11. Abdi AH, Luong C, Tsang T, Allan G, Nouranian S, Jue J, et al. Automatic Quality Assessment of Echocardiograms Using Convolutional Neural Networks: Feasibility on the Apical Four-Chamber View. IEEE Trans Med Imaging. 2017;36(6):1221-30. Epub 2017/04/10. doi:

10.1109/TMI.2017.2690836. PubMed PMID: 28391191. 
12. Madani A, Arnaout R, Mofrad M, Arnaout R. Fast and accurate view classification of echocardiograms using deep learning. NPJ Digit Med. 2018;1. Epub 2018/01/01. doi: 10.1038/s41746-017-0013-1. PubMed PMID: 30828647; PubMed Central PMCID: PMCPMC6395045.

13. Cannesson M, Tanabe M, Suffoletto MS, McNamara DM, Madan S, Lacomis JM, et al. A novel twodimensional echocardiographic image analysis system using artificial intelligence-learned pattern recognition for rapid automated ejection fraction. J Am Coll Cardiol. 2007;49(2):217-26. Epub 2007/01/16. doi: 10.1016/j.jacc.2006.08.045. PubMed PMID: 17222733.

14. Knackstedt C, Bekkers SC, Schummers G, Schreckenberg M, Muraru D, Badano LP, et al. Fully Automated Versus Standard Tracking of Left Ventricular Ejection Fraction and Longitudinal Strain: The FAST-EFs Multicenter Study. J Am Coll Cardiol. 2015;66(13):1456-66. Epub 2015/09/26. doi: 10.1016/j.jacc.2015.07.052. PubMed PMID: 26403342.

15. Tsang W, Salgo IS, Medvedofsky D, Takeuchi M, Prater D, Weinert L, et al. Transthoracic 3D Echocardiographic Left Heart Chamber Quantification Using an Automated Adaptive Analytics Algorithm. JACC Cardiovasc Imaging. 2016;9(7):769-82. Epub 2016/06/20. doi: 10.1016/j.jcmg.2015.12.020. PubMed PMID: 27318718.

16. Levy F, Dan Schouver E, lacuzio L, Civaia F, Rusek S, Dommerc C, et al. Performance of new automated transthoracic three-dimensional echocardiographic software for left ventricular volumes and function assessment in routine clinical practice: Comparison with 3 Tesla cardiac magnetic resonance. Arch Cardiovasc Dis. 2017;110(11):580-9. Epub 2017/06/02. doi: 10.1016/j.acvd.2016.12.015. PubMed PMID: 28566200.

17. Narang A, Mor-Avi V, Prado A, Volpato V, Prater D, Tamborini G, et al. Machine learning based automated dynamic quantification of left heart chamber volumes. Eur Heart J Cardiovasc Imaging. 2019;20(5):541-9. Epub 2018/10/12. doi: 10.1093/ehjci/jey137. PubMed PMID: 30304500; PubMed Central PMCID: PMCPMC6933871.

18. Genovese D, Rashedi N, Weinert L, Narang A, Addetia K, Patel AR, et al. Machine Learning-Based Three-Dimensional Echocardiographic Quantification of Right Ventricular Size and Function: Validation Against Cardiac Magnetic Resonance. J Am Soc Echocardiogr. 2019;32(8):969-77. Epub 2019/06/09. doi: 10.1016/j.echo.2019.04.001. PubMed PMID: 31174940.

19. Thavendiranathan P, Liu S, Datta S, Walls M, Nitinunu A, Van Houten T, et al. Automated quantification of mitral inflow and aortic outflow stroke volumes by three-dimensional real-time volume color-flow Doppler transthoracic echocardiography: comparison with pulsed-wave Doppler and cardiac magnetic resonance imaging. J Am Soc Echocardiogr. 2012;25(1):56-65. Epub 2011/11/23. doi: 10.1016/j.echo.2011.10.004. PubMed PMID: 22105057.

20. Jin CN, Salgo IS, Schneider RJ, Kam KK, Chi WK, So CY, et al. Using Anatomic Intelligence to Localize Mitral Valve Prolapse on Three-Dimensional Echocardiography. J Am Soc Echocardiogr. 2016;29(10):938-45. Epub 2016/08/23. doi: 10.1016/j.echo.2016.07.002. PubMed PMID: 27545445.

21. Aquila I, Fernandez-Golfin C, Rincon LM, Gonzalez A, Garcia Martin A, Hinojar R, et al. Fully automated software for mitral annulus evaluation in chronic mitral regurgitation by 3-dimensional 
transesophageal echocardiography. Medicine (Baltimore). 2016;95(49):e5387. Epub 2016/12/09. doi: 10.1097/MD.0000000000005387. PubMed PMID: 27930514; PubMed Central PMCID: PMCPMC5265986.

22. Prihadi EA, van Rosendael PJ, Vollema EM, Bax JJ, Delgado V, Ajmone Marsan N. Feasibility, Accuracy, and Reproducibility of Aortic Annular and Root Sizing for Transcatheter Aortic Valve Replacement Using Novel Automated Three-Dimensional Echocardiographic Software: Comparison with Multi-Detector Row Computed Tomography. J Am Soc Echocardiogr. 2018;31(4):505-14 e3. Epub 2017/11/28. doi: 10.1016/j.echo.2017.10.003. PubMed PMID: 29174341.

23. Narula S, Shameer K, Salem Omar AM, Dudley JT, Sengupta PP. Machine-Learning Algorithms to Automate Morphological and Functional Assessments in 2D Echocardiography. J Am Coll Cardiol. 2016;68(21):2287-95. Epub 2016/11/26. doi: 10.1016/j.jacc.2016.08.062. PubMed PMID: 27884247.

24. Mahmoud A, Bansal M, Sengupta PP. New Cardiac Imaging Algorithms to Diagnose Constrictive Pericarditis Versus Restrictive Cardiomyopathy. Curr Cardiol Rep. 2017;19(5):43. Epub 2017/04/14. doi: 10.1007/s11886-017-0851-0. PubMed PMID: 28405937.

25. Vidya KS, Ng EY, Acharya UR, Chou SM, Tan RS, Ghista DN. Computer-aided diagnosis of Myocardial Infarction using ultrasound images with DWT, GLCM and HOS methods: A comparative study. Comput Biol Med. 2015;62:86-93. Epub 2015/04/29. doi: 10.1016/j.compbiomed.2015.03.033. PubMed PMID: 25912990.

26. Sudarshan VK, Acharya UR, Ng EY, Tan RS, Chou SM, Ghista DN. An integrated index for automated detection of infarcted myocardium from cross-sectional echocardiograms using texton-based features (Part 1). Comput Biol Med. 2016;71:231-40. Epub 2016/02/24. doi: 10.1016/j.compbiomed.2016.01.028. PubMed PMID: 26898671.

27. Guo Y, Du GQ, Xue JY, Xia R, Wang YH. A novel myocardium segmentation approach based on neutrosophic active contour model. Comput Methods Programs Biomed. 2017;142:109-16. Epub 2017/03/23. doi: 10.1016/j.cmpb.2017.02.020. PubMed PMID: 28325439.

28. Buda M, Wildman-Tobriner B, Hoang JK, Thayer D, Tessler FN, Middleton WD, et al. Management of Thyroid Nodules Seen on US Images: Deep Learning May Match Performance of Radiologists. Radiology. 2019;292(3):695-701. Epub 2019/07/10. doi: 10.1148/radiol.2019181343. PubMed PMID: 31287391.

29. Chiao J-Y, Chen K-Y, Liao KY-K, Hsieh P-H, Zhang G, Huang T-C. Detection and classification the breast tumors using mask R-CNN on sonograms. Medicine. 2019;98(19). doi: 10.1097/md.0000000000015200.

30. Choi JS, Han BK, Ko ES, Bae JM, Ko EY, Song SH, et al. Effect of a Deep Learning Framework-Based Computer-Aided Diagnosis System on the Diagnostic Performance of Radiologists in Differentiating between Malignant and Benign Masses on Breast Ultrasonography. Korean J Radiol. 2019;20(5):74958. Epub 2019/04/18. doi: 10.3348/kjr.2018.0530. PubMed PMID: 30993926; PubMed Central PMCID: PMCPMC6470083. 
31. Han SS, Moon IJ, Lim W, Suh IS, Lee SY, Na Jl, et al. Keratinocytic Skin Cancer Detection on the Face Using Region-Based Convolutional Neural Network. JAMA Dermatol. 2019. Epub 2019/12/05. doi: 10.1001/jamadermatol.2019.3807. PubMed PMID: 31799995; PubMed Central PMCID: PMCPMC6902187.

32. Lang RM, Badano LP, Mor-Avi V, Afilalo J, Armstrong A, Ernande L, et al. Recommendations for cardiac chamber quantification by echocardiography in adults: an update from the American Society of Echocardiography and the European Association of Cardiovascular Imaging. J Am Soc Echocardiogr. 2015;28(1):1-39 e14. Epub 2015/01/07. doi: 10.1016/j.echo.2014.10.003. PubMed PMID: 25559473.

33. Main ML, Hannen MN, Kusnetzky LL, Martin JL, Coggins TR, Lanza P, et al. Myocardial contrast echocardiographic estimates of infarct size predict likelihood of left ventricular remodeling after acute anterior wall myocardial infarction. J Am Soc Echocardiogr. 2006;19(1):64-70. Epub 2006/01/21. doi: 10.1016/j.echo.2005.05.009. PubMed PMID: 16423671.

\section{Tables}

Table 1 Defining agreement using weighted kappa values

\begin{tabular}{|ll|}
\hline$k$ value & Agreement intensity \\
\hline$\times 0.2$ & poor \\
\hline $0.21-0.40$ & normal \\
$0.41-0.60$ & moderate \\
\hline $0.61-0.80$ & fairly strong \\
\hline $0.81-1.00$ & strong \\
\hline
\end{tabular}

Table 2 Performance comparison between the deep learning model and radiologists 


\begin{tabular}{|c|c|c|c|c|}
\hline Operator & $\begin{array}{l}\text { Sensitivity } \\
(\%)\end{array}$ & $\begin{array}{l}\text { Specificity } \\
(\%)\end{array}$ & AUC & $\begin{array}{l}\text { Experience } \\
\text { (years) }\end{array}$ \\
\hline Radiologist 1 & 53.33 & 83.33 & $\begin{array}{l}0.68(0.63- \\
0.74)\end{array}$ & 1 \\
\hline Radiologist 2 & 50.00 & 85.37 & $\begin{array}{l}0.68(0.62- \\
0.73)\end{array}$ & 2 \\
\hline Radiologist 3 & 56.67 & 81.30 & $\begin{array}{l}0.69(0.63- \\
0.74)\end{array}$ & 3 \\
\hline Radiologist 4 & 86.67 & 89.84 & $\begin{array}{l}0.88(0.84- \\
0.92)\end{array}$ & 10 \\
\hline Radiologist 5 & 83.33 & 91.06 & $\begin{array}{l}0.87(0.83- \\
0.91)\end{array}$ & 13 \\
\hline Radiologist 6 & 93.33 & 92.68 & $\begin{array}{l}0.93(0.89- \\
0.96)\end{array}$ & 15 \\
\hline $\begin{array}{l}\text { Average for junior } \\
\text { radiologists }\end{array}$ & 53.33 & 83.33 & $\begin{array}{l}0.68(0.63- \\
0.74)\end{array}$ & 2 \\
\hline $\begin{array}{l}\text { Average for senior } \\
\text { radiologists }\end{array}$ & 86.67 & 91.46 & $\begin{array}{l}0.89(0.85- \\
0.93)\end{array}$ & 13 \\
\hline Deep learning model & 83.33 & 95.53 & $\begin{array}{l}0.89(0.85- \\
0.93)\end{array}$ & NA \\
\hline
\end{tabular}

Data in parentheses are $95 \%$ confidence intervals. AUC = area under the receiver operating characteristic curve, NA = not applicable

Table 3 Comparison of average analysis duration per rat

\begin{tabular}{|c|c|c|}
\hline Observers & Time (sec) & $p$ \\
\hline Average for junior radiologists & $342(314-366)$ & $\varangle 0.001$ \\
\hline Average for senior radiologists & $223(193-235)$ & $\varangle 0.001$ \\
\hline Deep learning model & $154(148-169)$ & $\nabla 0.001$ \\
\hline
\end{tabular}

Data are median, data in parentheses indicate interquartile range (IQR).

Table 4 Interobserver agreement level 


\begin{tabular}{|lll|}
\hline Observers & $k$ & $95 \% \mathrm{Cl}$ \\
\hline Between senior radiologists & 0.86 & $0.82-0.94$ \\
\hline Between junior radiologists & 0.81 & $0.79-0.83$ \\
\hline Between all radiologists & 0.72 & $0.65-0.79$ \\
\hline Between deep learning model analyses & 0.96 & $0.92-0.99$ \\
\hline
\end{tabular}

Cl囚confidence interval

\section{Figures}
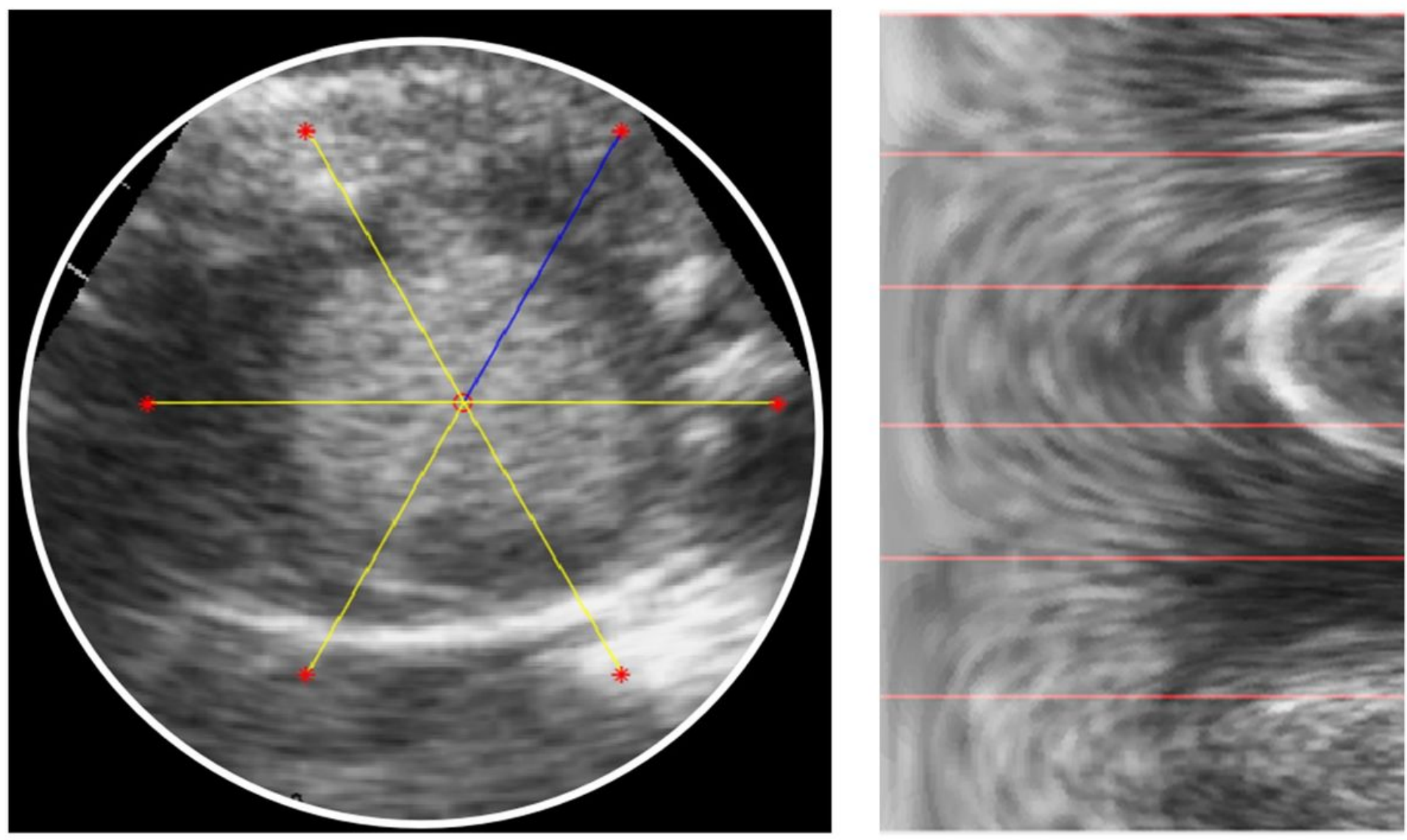

Figure 1

Representative example of patch images from a PROI image. The axial myocardium was divided into six segments starting from the blue line (1 o'clock position), each spanning 60 degrees. 


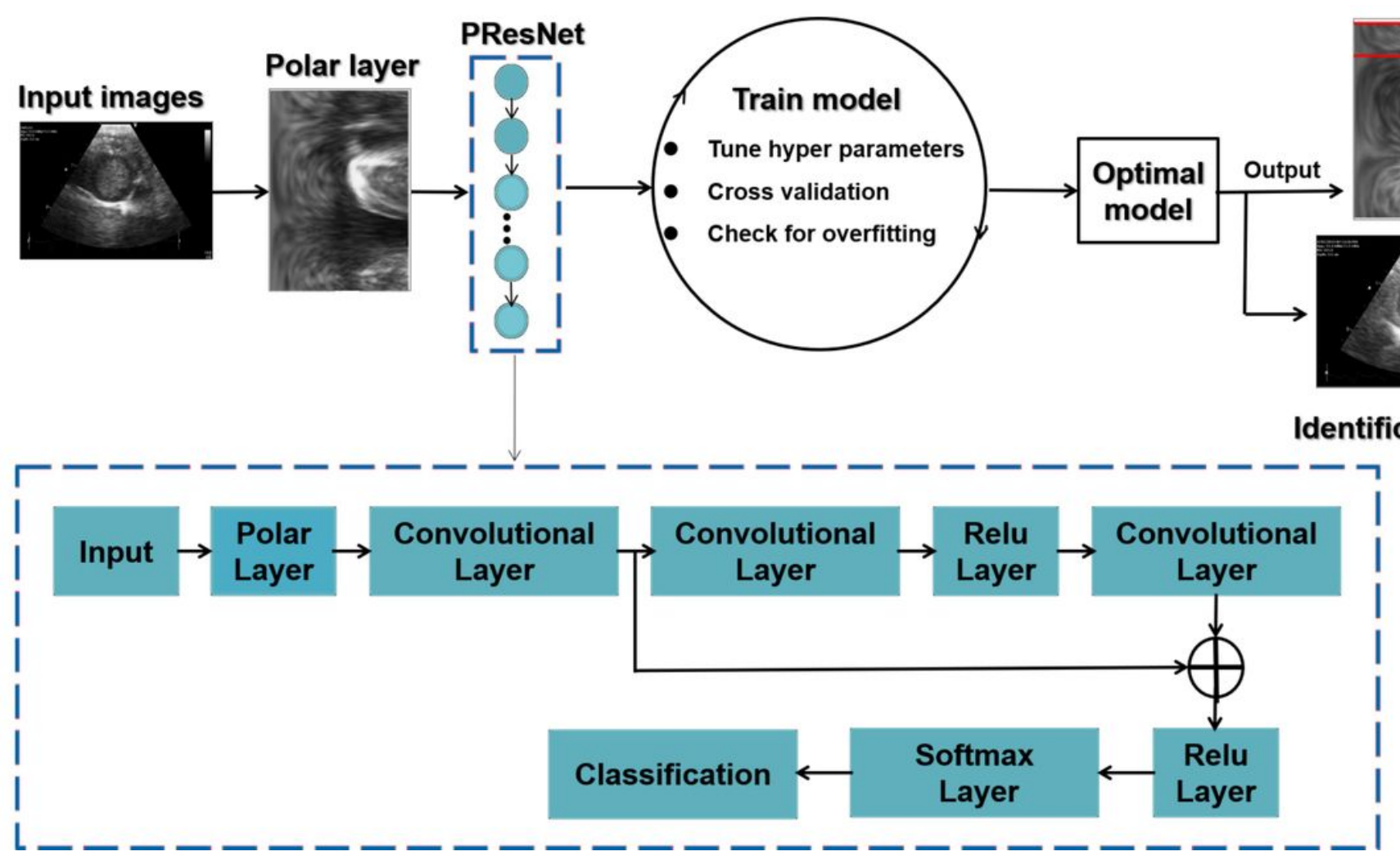

Figure 2

Flowchart of the utilized method.

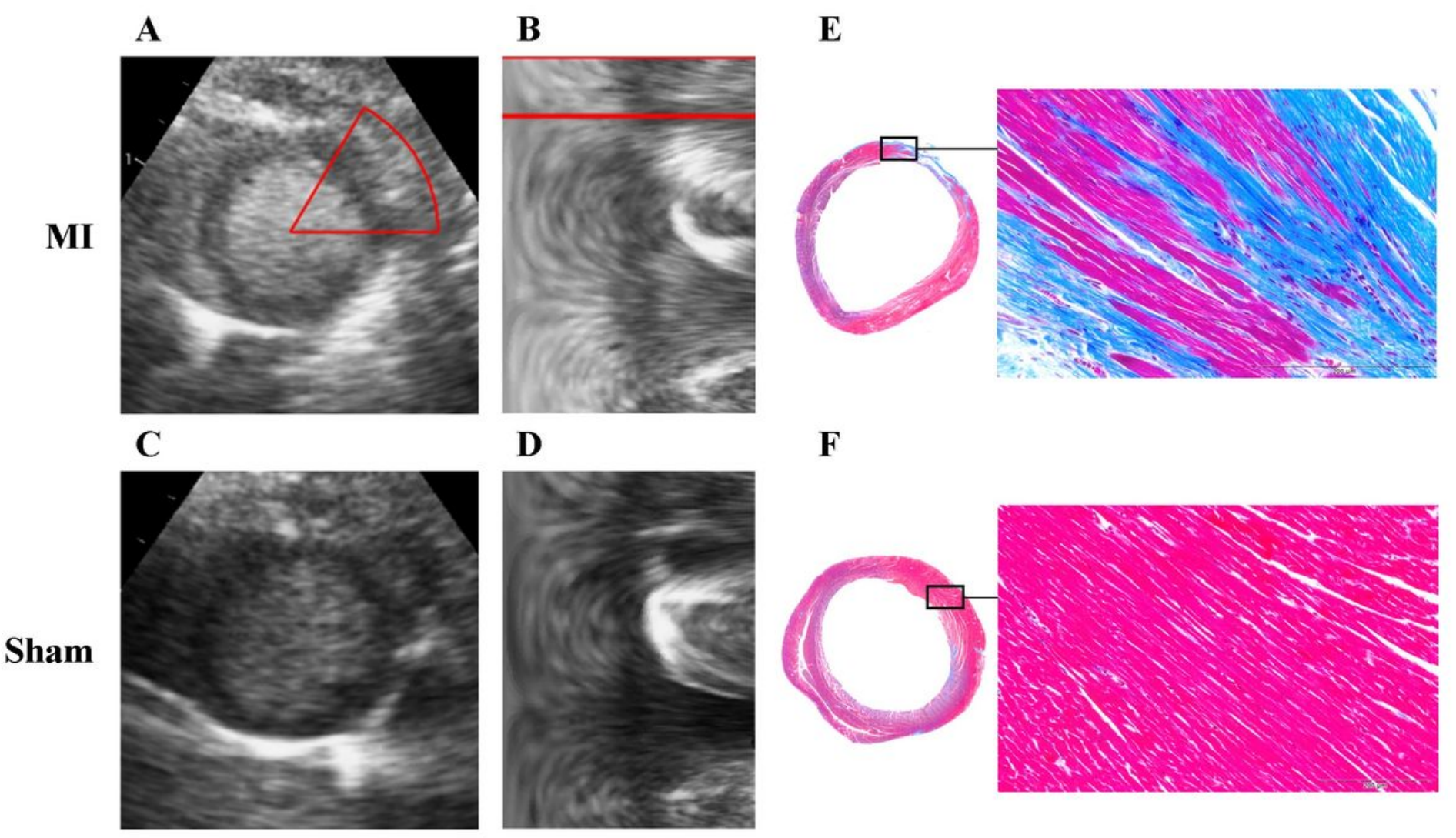

Figure 3 
MI detection using the deep learning model and histopathological staining. Deep learning model marking of an infarct segment (A, B) and non-marking of a non-infarct segment (C, D) in the short-axis of the myocardium and the polar layer. Representative photomicrographs (scale bar $=200 \mu \mathrm{m}$ ) of whole sectioned hearts and mid-papillary transverse sections stained with Masson's trichrome $(E, F)$ to depict scar areas at 28 days after ligation.

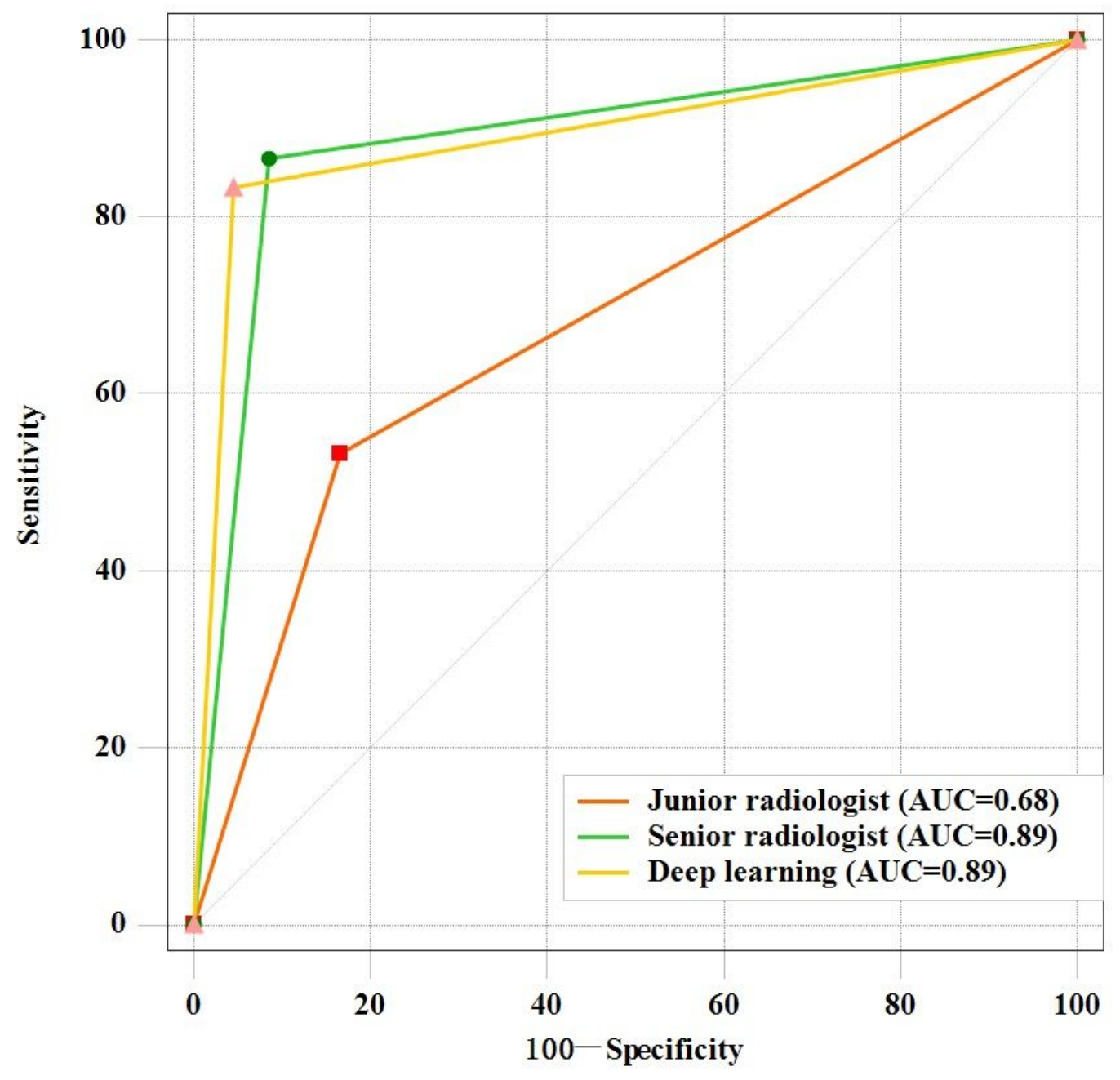

Figure 4

ROC curves for the deep learning model and six radiologists. Areas under the receiver operating characteristic curves (AUCs) for deep learning evaluation of 276 myocardial segments compared with the evaluations of six radiologists (three junior radiologists and three senior radiologists). AUC $=$ area under curve, $\mathrm{ROC}=$ receiver operating characteristic 


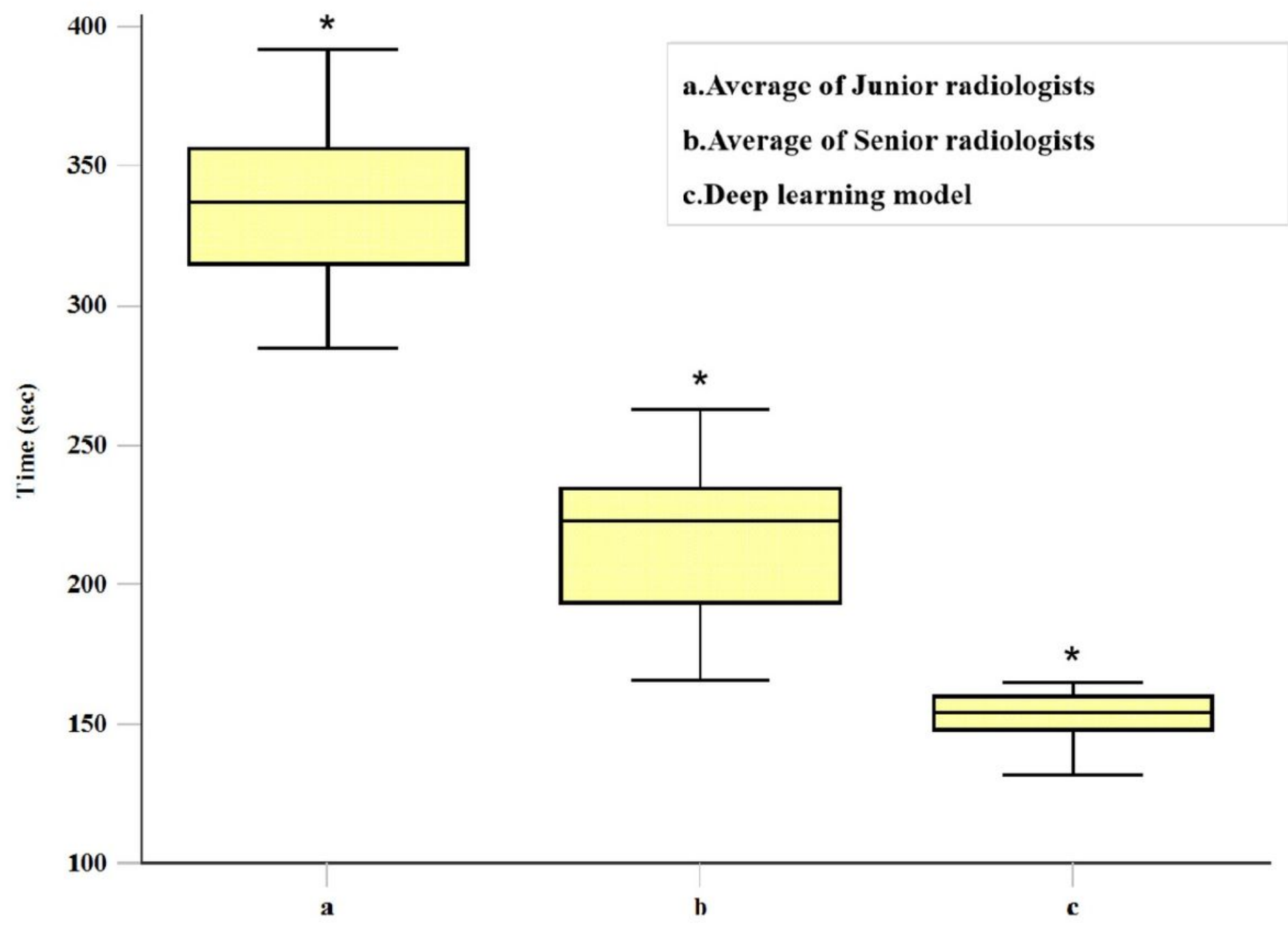

Figure 5

Box and whisker plots for analysis duration. Box and whisker plots to present MCE image analysis

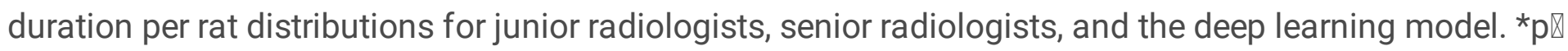
0.001 compared to any of the other groups. 